\title{
Влияние легирования ванадием на термоэлектрические свойства сплавов Гейслера $\mathrm{Fe}_{2} \mathrm{Ti}_{1-x} \mathrm{~V}_{x} \mathrm{Sn}$
}

\author{
(C) А.И. Таранова ${ }^{1}$, А.П. Новицкий ${ }^{1}$, А.И. Воронин ${ }^{1}$, С.В. Таскаев ${ }^{2,3}$, В.В. Ховайло \\ ${ }^{1}$ Национальный исследовательский технологический университет „МИСиС“, \\ 119049 Москва, Россия \\ 2 Челябинский государственный университет, \\ 454001 Челябинск, Россия \\ ${ }^{3}$ Южно-Уральский государственный университет, \\ 454080 Челябинск, Россия \\ E-mail: ataranovamisis@gmail.com
}

Поступила в Редакцию 7 февраля 2019 г.

В окончательной редакции 10 февраля 2019 г.

Принята к публикации 14 февраля 2019 г.

Представлены результаты экспериментального исследования сплавов $\mathrm{Fe}_{2} \mathrm{Ti}_{1-x} \mathrm{~V}_{x} \mathrm{Sn}(x=0,0.06,0.15,0.2)$. По полученным температурным зависимостям электрической проводимости, коэффициента термоэдс и теплопроводности установлено, что исследуемые составы проявляют транспортные свойства, типичные для полупроводников, а частичное замещение атомов титана атомами ванадия приводит к смене дырочного типа проводимости на электронный, наилучшими термоэлектрическими характеристиками обладает нелегированный образец $\mathrm{Fe}_{2} \mathrm{TiSn}$.

DOI: 10.21883/FTP.2019.06.47727.36

\section{1. Введение}

Полные сплавы Гейслера представляют собой тройные интерметаллические или металлические соединения с общей формулой $\mathrm{X}_{2} \mathrm{YZ}$, где $\mathrm{X}, \mathrm{Y}$ - переходные $3 d$ - или $4 d$-элементы или редкоземельные металлы, а $\mathrm{Z}$ - металлоиды. Структурный тип кристаллической решетки $L 2_{1}$, в центре куба позиция занята атомом $\mathrm{X}$, тогда как Z и $\mathrm{Y}$ поочередно располагаются в узлах решетки [1]. Элементарная ячейка содержит 24 валентных электрона, изменяя количество которых, можно управлять термоэлектрическими параметрами [2].

Из-за того что сплавы Гейслера термически стабильны, обладают высокой электропроводностью и довольно большим коэффициентом термоэдс, а элементы, входящие в их состав, доступны, среди низко- и среднетемпературных термоэлектрических материалов их можно рассматривать как наиболее перспективные [3].

Наиболее изученными в данном классе являются соединения на основе $\mathrm{Fe}_{2} \mathrm{VAl}$, коэффициент термоэдс в которых при комнатной температуре достигает лишь 35 мкВ/K, из-за чего показатель термоэлектрической добротности ZT не превышает 0.3 в соединениях $n$ - и p-типа проводимости $[4,5]$. Как и $\mathrm{Fe}_{2} \mathrm{VAl}$, интерметаллический сплав состава $\mathrm{Fe}_{2} \mathrm{TiSn}$ обладает кристаллической структурой типа $L 2_{1}$, в которой магнитные и электрические свойства значительно зависят от положения атомов железа.

Согласно теоретическим расчетам, при увеличении числа валентных электронов для $\mathrm{Fe}_{2} \mathrm{TiSn}$ с 24 до 24.06 фактор мощности достигает максимального значения по сравнению с нелегированным образцом [6]. Теоретические расчеты [6] предсказывают, что частичная замена атомов титана на ванадий или другие элементы со схожей электронной конфигурацией приводит к росту термоэдс и фактора мощности за счет внесения дополнительного электрона в систему в расчете на элементарную ячейку. С целью проверки теоретических предсказаний в данной работе были исследованы термоэлектрические свойства серии образцов состава $\mathrm{Fe}_{2} \mathrm{Ti}_{1-x} \mathrm{~V}_{x} \mathrm{Sn}(x=0,0.06,0.15,0.2)$.

\section{2. Образцы и методы исследования}

Образцы химического состава $\mathrm{Fe}_{2} \mathrm{Ti}_{1-x} \mathrm{~V}_{x} \mathrm{Sn}(x=0$, $0.06,0.15,0.2)$ были получены методом электродуговой плавки на установке 5 Bell Jar в инертной атмосфере аргона. В качестве прекурсоров в соответствии со стехиометрическим соотношением были использованы $\mathrm{Fe}, \mathrm{Ti}, \mathrm{V}$ и $\mathrm{Sn}$. Для гомогенизации состава после плавки слитки были завернуты в танталовую фольгу и помещены в кварцевую ампулу, которая подвергалась отжигу при температуре $1073 \mathrm{~K}$ в течение одной недели в вакууме, после чего была проведена закалка погружением в воду [7]. Отожженные слитки были измельчены в механической ступке Fritsch Pulverisette 2, затем спекались на установке Labox 650, Sinter Land при температуре $1073 \mathrm{~K}$, давлении $50 \mathrm{MПа,} \mathrm{скорости}$ нагрева $100 \mathrm{~K} /$ мин в вакууме. После консолидации полученные образцы отжигались при температуре $1073 \mathrm{~K}$ в течение 10 ч в вакууме.

Анализ фазового состава исследуемых образцов проводился на дифрактометре Дифрей 401 с использованием $\mathrm{Cr} K_{\alpha}$-излучения (длина волны $\lambda=2.2937 \AA$ ). Методом термогравиметрического анализа на термоанализаторе Simultaneous Differential Technics (SDT Q600) 
была исследована термическая стабильность исследуемых составов в условиях линейного нагрева со скоростью $10 \mathrm{~K} /$ мин в интервале температур 298-1173 K в атмосфере аргона. Для качественного элементного анализа был использован сканирующий электронный микроскоп (СЭМ) Tescan Vega 3SB с приставкой для энергодисперсионной рентгеновской спектроскопии (EDX).

Теплопроводность $\kappa$ определялась из измерений температурной зависимости температуропроводности в соответствии с формулой $\kappa=C_{p} d \chi$, где $C_{p}$ - удельная теплоемкость, $d$ - объемная плотность, определяемая методом гидростатического взвешивания, $\chi-$ коэффициент температуропроводности. Коэффициент температуропроводности был измерен на установке Netzsch LFA 447 методом лазерной вспышки в температурном интервале 300-573 К. Температурные зависимости электропроводности и коэффициента термоэдс определялись в диапазоне температур 80-973 K четырехзондовым и дифференциальным методами соответственно.

\section{3. Результаты исследований}

Результаты рентгенофазового анализа образцов состава $\mathrm{Fe}_{2} \mathrm{Ti}_{1-x} \mathrm{~V}_{x} \mathrm{Sn}(x=0,0.06,0.15,0.2)$ после консолидации и отжига представлены на рис. 1. Основная часть пиков по межплоскостным расстояниям, интенсивностям, а также углам отражения совпадает с соответствующими характеристиками для $\mathrm{Fe}_{2} \mathrm{TiSn}$, имеющего кубическую решетку с периодом $a=6.0680 \AA[8]$, примесные фазы обнаружены не были.

Согласно проведенному термогравиметрическому анализу, все исследуемые образцы термически и химически стабильны до температуры $1173 \mathrm{~K}$, активно протекающих процессов окисления или восстановления не наблюдается, потери массы незначительны $(<0.5 \%)$.

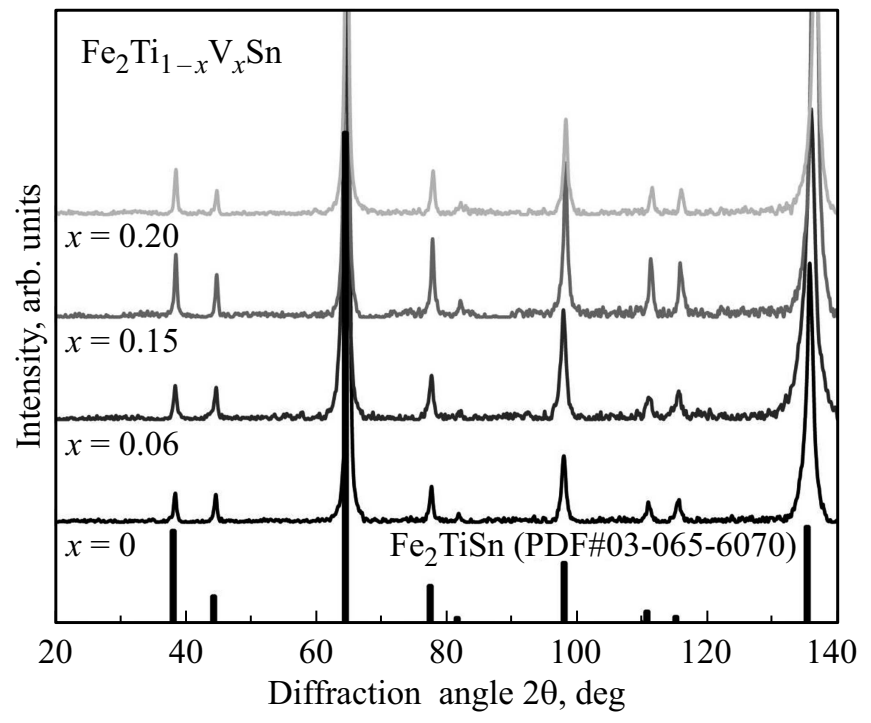

Рис. 1. Дифрактограммы образцов $\mathrm{Fe}_{2} \mathrm{Ti}_{1-x} \mathrm{~V}_{x} \mathrm{Sn}(x=0,0.06$, $0.15,0.2)$ после консолидации и отжига.

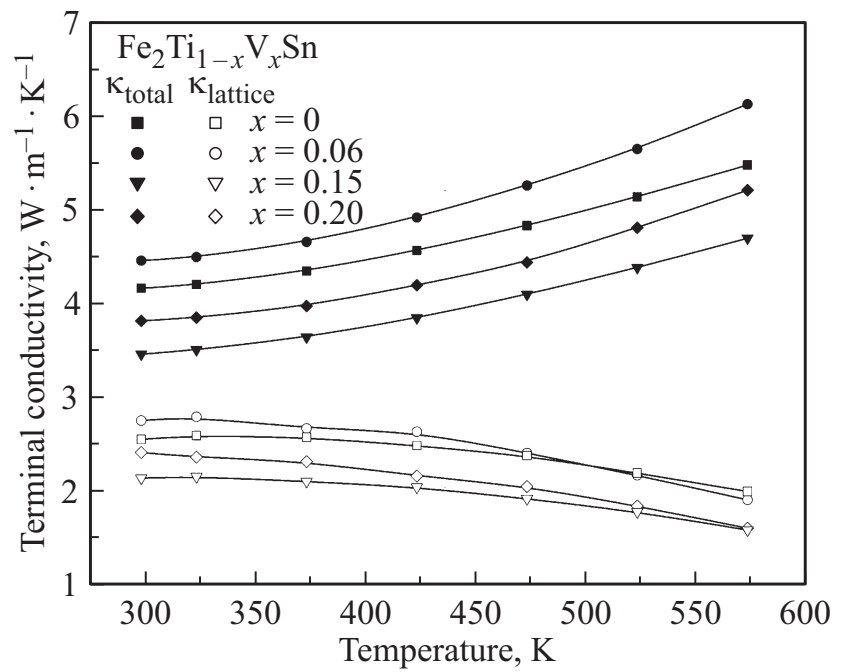

Рис. 2. Температурная зависимость общей и решеточной теплопроводностей образцов $\mathrm{Fe}_{2} \mathrm{Ti}_{1-x} \mathrm{~V}_{x} \mathrm{Sn}(x=0,0.06,0.15,0.2)$.

По результатам энергодисперсионного анализа во всех образцах $\mathrm{Fe}_{2} \mathrm{Ti}_{1-x} \mathrm{~V}_{x} \mathrm{Sn}(x=0,0.06,0.15,0.2)$ обнаружены области, обогащенные титаном и оловом, а по границам зерен установлено наличие оксида титана, что может быть вызвано неэффективным протеканием реакции в процессе плавки. Также полученный результат может быть обусловлен достаточной узкой областью гомогенности соединения $\mathrm{Fe}_{2} \mathrm{TiSn}$. При исследовании фазовых равновесий системы $\mathrm{Fe}-\mathrm{Ti}-\mathrm{Sn}$ было установлено, что соединение состава $\mathrm{Fe}_{2} \mathrm{TiSn}$ чувствительно к соотношению железа и титана и становится нестабильным при небольшом отклонении содержания олова от стехиометрического [9].

На рис. 2 представлены температурные зависимости общей $\left(\kappa_{\text {total }}\right)$ и решеточной $\left(\kappa_{\text {lattice }}\right)$ теплопроводности сплавов $\mathrm{Fe}_{2} \mathrm{Ti}_{1-x} \mathrm{~V}_{x} \mathrm{Sn}(x=0,0.06,0.15,0.2)$. В соответствии с полученными данными максимальное значение теплопроводности отвечает образцу номинального состава $\mathrm{Fe}_{2} \mathrm{Ti}_{0.94} \mathrm{~V}_{0.06} \mathrm{Sn}$, который также обладает и максимальной плотностью $d\left(d / d_{\text {theor }}=92 \%, d_{\text {theor }}-\right.$ теоретическое значение), в то время как наиболее пористому образцу исследуемой серии $\mathrm{Fe}_{2} \mathrm{Ti}_{0.85} \mathrm{~V}_{0.15} \mathrm{Sn}$ $\left(d / d_{\text {theor }}=86 \%\right)$ соответствует минимальная теплопроводность, $\sim 3.47 \mathrm{BT} \cdot \mathrm{M}^{-1} \cdot \mathrm{K}^{-1}$ (при комнатной температуре). Общая теплопроводность сплавов $\mathrm{Fe}_{2} \mathrm{Ti}_{1-x} \mathrm{~V}_{x} \mathrm{Sn}$ монотонно растет при повышении температуры (рис. 2). Полученные результаты указывают на то, что с ростом температуры происходит смена превалирующей составляющей теплопроводности с решеточной на электронную. Решеточная теплопроводность во всем температурном диапазоне монотонно убывает, однако это изменение невелико $\left(\lesssim 0.5 \mathrm{BT} \cdot \mathrm{M}^{-1} \cdot \mathrm{K}^{-1}\right)$, что можно объяснить близкими значениями атомных радиусов титана $(1.47 \AA)$ и ванадия $(1.34 \AA)$.

В соответствии с законом Видемана-Франца характер полученной температурной зависимости электропровод- 


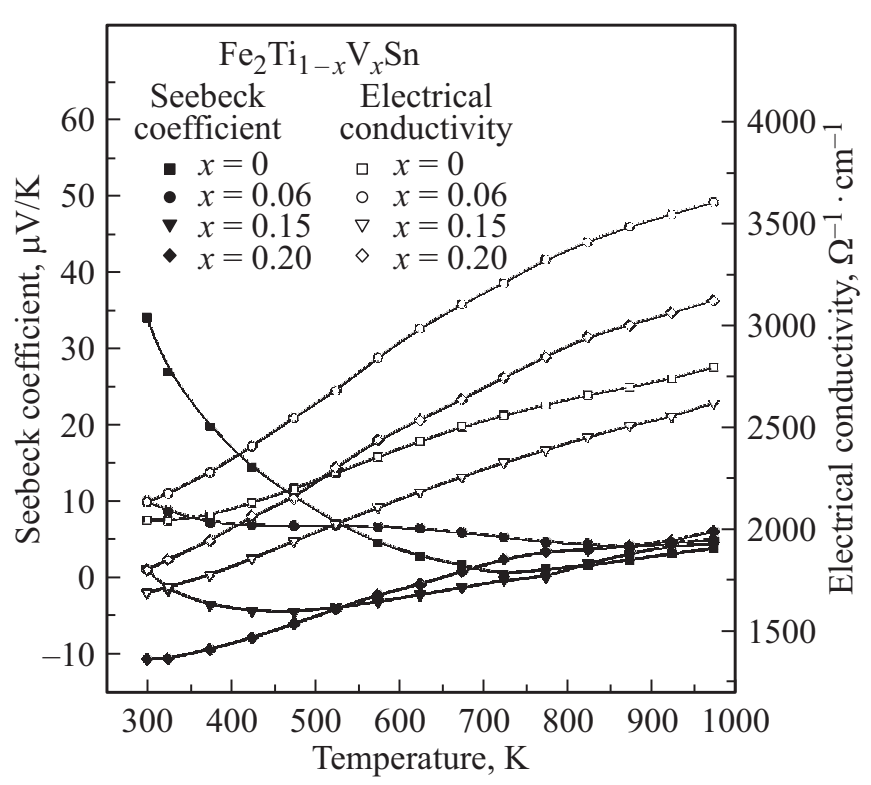

Рис. 3. Температурные зависимости коэффициента термоэдс и электропроводности образцов $\mathrm{Fe}_{2} \mathrm{Ti}_{1-x} \mathrm{~V}_{x} \mathrm{Sn}(x=0,0.06$, $0.15,0.2)$.

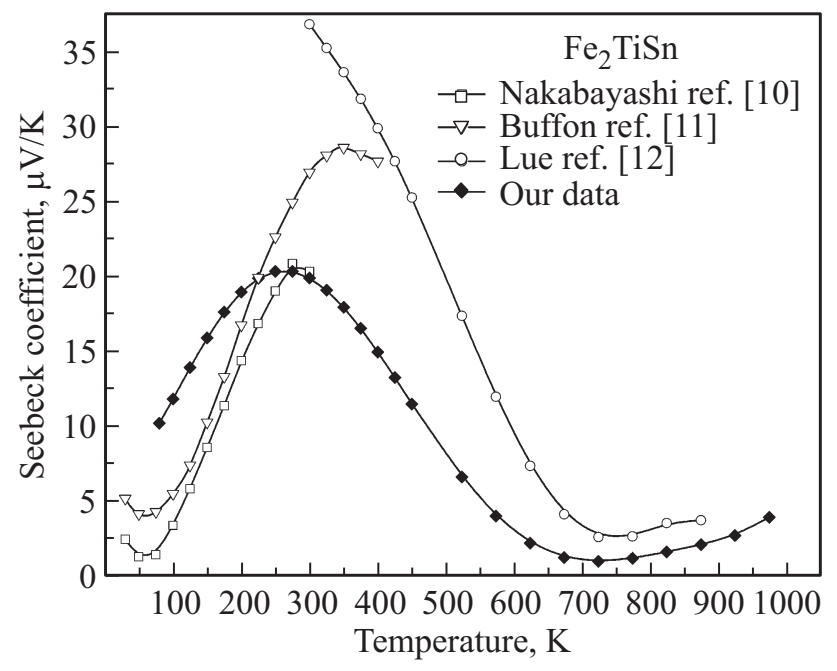

Рис. 4. Сравнение температурной зависимости коэффициента термоэдс для $\mathrm{Fe}_{2} \mathrm{TiSn}$.

ности полностью соответствует значениям теплопроводности для всех образцов. Из рис. 3 следует, что исследуемые сплавы относятся к полупроводниковому типу материалов, так как при повышении температуры значение электропроводности также возрастает. Максимальное значение коэффициента термоэдс наблюдается при температуре $300 \mathrm{~K}$ для нелегированного образца $\mathrm{Fe}_{2} \mathrm{TiSn}$ и составляет $35 \mathrm{m \kappa B} / \mathrm{K}$, что на порядок меньше заявленного теоретического значения [6]. При частичном замещении титана ванадием с последующим увеличением концентрации последнего происходит смена доминирующего типа проводимости с дырочного на электронный.
С целью сравнения полученных температурных зависимостей с литературными данными для образца состава $\mathrm{Fe}_{2} \mathrm{TiSn}$ коэффициент термоэдс был измерен в более широком температурном интервале - от 80 до $973 \mathrm{~K}$. На рис. 4 приведена температурная зависимость коэффициента термоэдс, измеренная в нашей работе; для сравнения показаны также результаты измерений термоэдс, опубликованные в работах других научных групп. Из сравнения этих данных видно, что результаты наших измерений хорошо коррелируют с исследованиями, опубликованными в работах [10-12].

Необходимо отметить, что в серии образцов $\mathrm{Fe}_{2} \mathrm{Ti}_{1-x} \mathrm{~V}_{x} \mathrm{Sn}$, которая была исследована, однозначной зависимости транспортных свойств от степени замещения $x$ не наблюдается. Можно предположить, что наблюдаемая немонотонная зависимость теплопроводности и электропроводности от уровня допирования обусловлена как наличием примесных фаз в образцах, так и различием в удельной плотности образцов. Несмотря на то что содержание примесных фаз не превышает 5\%, наличие примесных фаз, обогащенных титаном и оловом, сильно влияет на термоэлектрические свойства исследуемой серии образцов.

\section{4. Заключение}

В работе показано, что теоретические предположения о повышении термоэлектрической эффективности сплава $\mathrm{Fe}_{2} \mathrm{TiSn}$ с помощью частичной замены атомов титана на ванадий не нашли экспериментального подтверждения. Значения коэффициента термоэдс, измеренные при комнатной температуре, имеют тенденцию к понижению при замещении титана на ванадий в образцах $\mathrm{Fe}_{2} \mathrm{Ti}_{1-x} \mathrm{~V}_{x} \mathrm{Sn}$, что негативно сказывается на значениях термоэлектрической эффективности ванадийсодержащих образцов. Максимальное значение коэффициента термоэдс, полученное экспериментально, составляет лишь $35 \mathrm{мкB} / \mathrm{K}$, что на порядок меньше теоретически рассчитанного.

Работа поддержана Российским фондом фундаментальных исследований (грант № 18-38-20170).

\section{Список литературы}

[1] I. Galanakis. In: Theory of Heusler and Full-Heusler Compounds, ed. by C. Felser, A. Hirohata [Heusler Alloys Properties, Growth, Applications, v. 222 (Springer Ser. Mater. Sci., 2013)].

[2] T. Graf, C. Felser, S.S.P. Parkin. Prog. Solid State Chem., 39 (1), 1 (2011).

[3] В.В. Ховайло, А.И. Воронин, В.Ю. Зуева, М.А. Середина, Р. Чаттерджи. ФТП, 51, 752 (2017).

[4] C.S. Lue, C.F. Chen, J.Y. Lin, Y.T. Yu, Y.K. Kuo. Phys. Rev. B, 75 (6), 064204 (2007).

[5] Y. Nishino, Y. Tamada. J. Appl. Phys., 115, 123707 (2014).

[6] S. Yabuuchi, M. Okamoto, A. Nishide, Y. Kurosaki, J. Hayakawa. Appl. Phys. Express, 6 (2), 025504 (2013). 
[7] A. Slebarski, M.B. Maple, E.J. Freeman, C. Sirvent, D. Tworuszka, M. Orzechowska, M. Neumann. Phys. Rev. B, 62 (5), 3296 (2010).

[8] Y. Fujita, K. Endo, M. Terada, R. Kimura. J. Phys. Chem. Solids, 33 (7-9), 1443 (1972).

[9] M. Yin, P. Nash, J.A. Kaduk, J.C. Schuster. J. Alloys Compd., 693, 76 (2017).

[10] M. Nakabayashi, K. Fukuda, H. Kitagawa, Y. Yamada, S. Kubo, A. Matsushita. Physica B, 329, 1134 (2003).

[11] M.L. Buffon, G. Laurita, L. Lamontagne, E.E. Levin, S. Mooraj, D.L. Lloyd, R. Seshadri. J. Phys.: Condens. Matter, 29 (40), 405702 (2017).

[12] C.S. Lue, Y.K. Kuo. J. Appl. Phys., 96 (5), 2681 (2004).

Редактор Л.В. Шаронова

\section{Influence of doping with $\mathbf{V}$ \\ on thermoelectric properties \\ of $\mathrm{Fe}_{\mathbf{2}} \mathrm{Ti}_{1-x} \mathrm{~V}_{x} \mathrm{Sn}$ Heusler alloys}

A.I. Taranova ${ }^{1}$, A.P. Novitskii ${ }^{1}$, A.I. Voronin ${ }^{1}$, S.V. Taskaev ${ }^{2,3}$, V.V. Khovaylo ${ }^{1}$

${ }^{1}$ National University of Science and Technology

„MISIS“,

119049 Moscow, Russia

${ }^{2}$ Chelyabinsk State University,

454001 Chelyabinsk, Russia

${ }^{3}$ South Ural State University,

454080 Chelyabinsk, Russia

Abstract In this work the results of experimental study of $\mathrm{Fe}_{2} \mathrm{Ti}_{1-x} \mathrm{~V}_{x} \mathrm{Sn}$ alloys $(x=0,0.06,0.15,0.2)$ are presented. According to the temperature dependencies of the electrical conductivity, Seebeck coefficient and thermal conductivity, it is established, that the studied compositions exhibit transport properties typical for semiconductors. The substitution of $\mathrm{Ti}$ at $\mathrm{V}$ leads to a change of the $p$-type electrical conductivity behavior to the $n$-type; the pristine sample of $\mathrm{Fe}_{2} \mathrm{TiSn}$ has the best thermoelectric characteristics. 